Acta Crystallographica Section D

Biological Crystallography

ISSN 0907-4449

\section{Elena V. Orlova}

Imperial College of Science Technology and Medicine, Department of Biochemistry, London SW7 2AY, England

Correspondence e-mail: e.orlova@ic.ac.uk

\title{
Structural analysis of non-crystalline macromolecules: the ribosome
}

Single-particle analysis using cryo-electron microscopy has emerged recently as a tool for elucidating the structure of biological macromolecules and their assemblies. A prerequisite for single-particle analysis is an ensemble of images of structurally identical particles in different orientations. There are a variety of techniques used for image processing of this type of object in electron microscopy. The paper gives an overview of the general philosophy of image analysis of single particles in electron microscopy. It has been shown that multivariate statistical analysis of large data sets in conjunction with angular reconstitution is capable of yielding a structural resolution approaching that of X-ray structural analysis of large macromolecules. Structure preservation during specimen preparation for electron microscopy is crucial for high-resolution studies. Nowadays, cryo-electron microscopy is a beneficial method for studying biological macromolecules in their natural environment, allowing their rapid freezing in a particular functional state. The processing of images of 50S Escherichia coli ribosomal subunits embedded in vitreous ice is used as an example of image analysis of single particles at $7.5 \AA$ resolution.

\section{Introduction}

$\mathrm{X}$-ray crystallography has been known for several decades as a well established technique of structural analysis at atomic resolution. Development of several novel approaches to phase determination have made this technique a powerful tool for solving the structures of large macromolecular complexes. However, a prerequisite for such structure determination is the availability of good crystals. NMR spectroscopy allows the study of molecular structure and dynamics in solution but can only be applied to a system with a relatively small mass. Cryoelectron microscopy is a relatively new tool that can be used in structural analysis of single molecules or of macromolecular assemblies ('single-particle analysis') with molecular masses ranging from several hundred kilodaltons (Akiba et al., 1996) to megadaltons (Chiu, 1993).

Electron microscopy (EM) has been used for the visualization of biological macromolecules for almost half a century. In the 1970s and 1980s, the negative-stain specimen-preparation technique was the main technique used for visualizing macromolecular assemblies by EM. The images of negatively stained molecules were typically interpreted visually and provided the first structural insights into macromolecules such as ribosomes. However, the information provided by an electron microscope is essentially two-dimensional (2D) and the three-dimensional (3D) structural interpretations of the $2 \mathrm{D}$ images were subjective.
Received 25 April 2000 Accepted 5 August 2000
(C) 2000 International Union of Crystallography Printed in Denmark - all rights reserved 
An important question in those days was how to obtain the true 3D density information of the molecules under investigation based on the 2D images obtained from the electron microscope. Considerable effort was invested in the understanding of the theory of image formation in the microscope. In the 1960s and 1970s, researchers in laboratories in the UK, the USA, Germany and Russia worked on the theory and practical algorithms for the digital reconstruction of 3D structures from 2D projections of an object (for a review, see Herman, 1980; Hoppe \& Hegerl, 1980).

Further developments in specimen-preparation techniques, especially the fast-freezing methods of what is now known as 'cryo-EM', gave new opportunities for studying macromolecules in their natural environment (Dubochet et al., 1982). They allowed the improvement of the resolution attainable in the images and the reconstruction of undistorted biological macromolecules owing to better structure preservation.

The ribosome, because of its biological significance, has long intrigued structural biologists. This very complicated assembly of RNAs and proteins has been difficult to analyse by X-ray crystallography because of its large size (2.5 MDa) and problems with crystallization. On the other hand, it was possible to observe ribosomes in the electron microscope and to make preliminary suggestions about the structure (Boublik et al., 1977). Visual comparison of single-particle images made it possible to suggest the arrangement of subunits within the complex. The interpretation of ribosomes embedded in heavymetal salts (for a review of negative-stain techniques, see Harris \& Horne, 1991) revealed the overall shape of the whole ribosome and its small and large subunits (Boublik et al., 1977; Lake, 1982).

The computational era of ribosome image analysis started in the 1980s with the use of alignments and classification (Frank et al., 1981, 1982; van Heel \& Frank, 1981; van Heel \& Stöffler-Meilicke, 1985). First 3D reconstructions were performed using images of negatively stained ribosomes (Frank et al., 1988; Radermacher, 1988; Radermacher et al., 1987). In these results, details up to $30 \AA$ could be distinguished. Unfortunately, the negative-stain technique has serious drawbacks: molecules are flattened and distorted by dehydration in the procedure of preparation, the molecules often exhibit preferential attachment to the supporting film, the resolution of obtained images is restricted by the size of grains of the negative stain and the molecules can be chemically modified by the stain. Images of a molecule also depend on the type of negative stain used. In the last decade, (cryo-) electron microscopy has become the main tool for structural analysis of macromolecules organized in small assemblies or 2D crystals (see Böttcher et al., 1997; Fujiyoshi, 1999; Henderson et al., 1986; Matadeen et al., 1999; Mitsuoka et al., 1999; Miyazawa et al., 1999; Nogales et al., 1997).

\section{Specimen preparation for electron microscopy}

The modern alternative to the use of dry negative stain as an embedding medium is the preparation of rapidly frozen hydrated biological specimens (Dubochet et al., 1982). Here, the sample is applied to a holey carbon film. After removing excess fluid by blotting, a thin layer of solution on an electron microscopic grid is obtained. The grid is then plunged into liquid propane or ethane. At a freezing rate of more than $10^{5} \mathrm{~K} \mathrm{~s}^{-1}$ the aqueous suspension converts to a vitreous phase directly, bypassing the crystalline ice. Thus, the frozen grid will have a thin aqueous layer with biological macromolecules embedded in an almost natural hydrated environment. After freezing, grids can be kept for a long time at liquid-nitrogen temperatures. These structurally preserved frozen-hydrated samples are then imaged in an electron microscope at liquidnitrogen temperatures (Dubochet et al., 1988).

Structural analysis with details smaller than $10 \AA$ is possible with the frozen specimens. However, such objects are highly sensitive to electron irradiation. In order to reduce radiation damage, images have to be taken at low illumination doses. In addition, it was found that low temperature reduces radiation damage (Luther et al., 1988). Specimen stability is significantly increased at liquid-nitrogen temperatures and is even better at liquid-helium temperatures (Lamvik, 1991; Stark et al., 1996). Thus, electron microscopes equipped with cryo specimen holders having liquid nitrogen or helium operating at accelerating voltages of $200-300 \mathrm{kV}$ can provide images for highresolution $3 \mathrm{D}$ analysis.

\section{Analysis of single-molecule images}

\subsection{Image restoration}

As soon as grids have been prepared with a specimen (ribosomes), images can be taken in an electron microscope. A beam of electrons irradiates the specimen (molecules within vitrified ice); electrons passing close to the specimen atoms are deflected by their electric fields (scattered). The magnetic lenses of the microscope then focus the deflected electrons so that a magnified image of the molecule will be formed in the plane of the micrograph. Biological objects such as ribosomes or any other macromolecules consist mainly of atoms with low atomic numbers such as $\mathrm{H}, \mathrm{O}, \mathrm{N}, \mathrm{P}, \mathrm{Ca}$ and $\mathrm{C}$. The objects are too thin to absorb the electrons from illuminating beam. These electrons are only weakly scattered by the specimens and change mainly their phase, not their amplitude. Such samples are called 'weak phase objects', since the observed images have phase contrast. It was found that images of such objects could be seen as a result of aberrations of the optical system of the electron microscope. The theory of image formation was developed at the end of the 1970s and in the 1980s (Frank, 1973; Glaeser, 1985; Hawkes, 1992; Saxton, 1978) and provided a way to interpret images. Having obtained images of ribosomes from the electron microscope (Fig. 1), it is necessary to correct for distortions caused by aberrations of the imaging system. The function describing the influence of the imaging system (in our case the electron microscope) on the resulting image is called the 'contrast-transfer function' (CTF; Hawkes, 1992; Saxton, 1978). A procedure for compensating these aberrations was named the image 'restoration', although 
nowadays it can be said that 'the images are corrected for the CTF' (Conway \& Steven, 1999; Erickson \& Klug, 1970; Ludtke et al., 1999; Penczek et al., 1994). A restored (corrected) image corresponds to a true projection of the specimen, although this approximation is valid only for a limited thickness of the sample. The higher the accelerating voltage of the microscope is, the thicker a specimen slice will become, the image of which will correspond to a projection at a given defocus.

\subsection{Enhancement of signal-to-noise ratio in the images}

Radiation sensitivity of the biological samples, low exposure doses (to prevent disintegration of molecules) and emulsion granularity all generate a high level of noise on images. Thus, the signal-to-noise ratio (SNR) in the images is very low. SNR is usually defined as the ratio of energies of the signal and noise. From statistical analysis, it is known for an additive noise that averaging will reduce the standard deviation of the noise in images by $K^{1 / 2}$, where $K$ is the number of averaged images (Cramer, 1946).

Molecules in the thin frozen layer in a specimen assume arbitrary orientations. The position of a molecule within the vitrified water layer can be described by three translational coordinates $x, y$ and $z$, where $x$ and $y$ are the coordinates within the plane of the grid and $z$ is the coordinate along the optical axis of the microscope. The latter is the projection direction of molecular densities in the microscope and hence

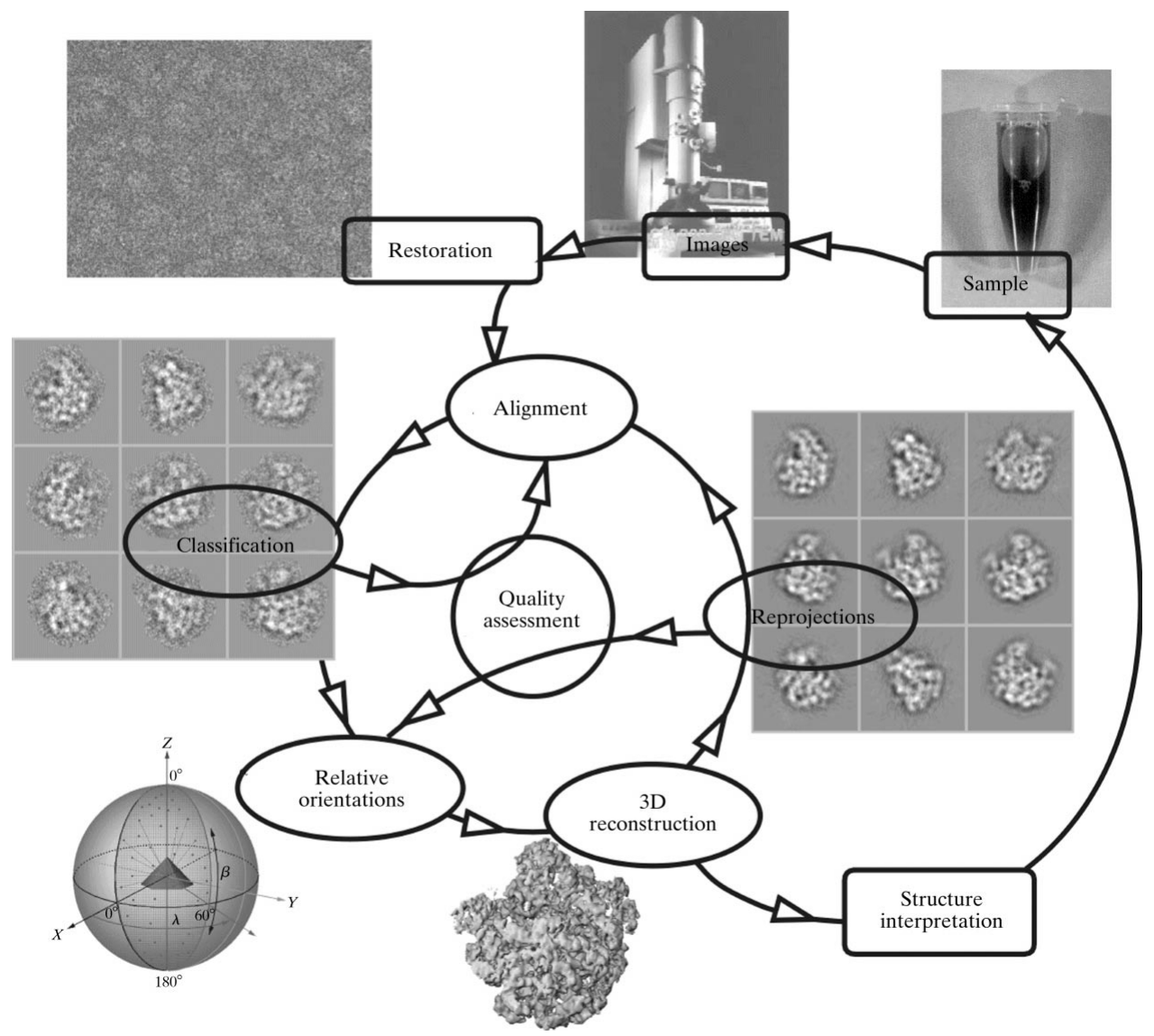

Figure 1

General scheme of image processing. does not relate to the orientation of the image. The orientation of a molecule within the layer can be described by the Euler angles $\alpha, \beta, \gamma$. In our definition, the angle $\alpha$ characterizes rotation of the image in the plane of the micrograph. The other two angles $(\beta, \gamma)$ describe rotations of the molecule out of the micrograph plane. Since the molecules have such a freedom in orientation and position, averaging of all images produces smearing of the densities. To enhance a signal (a distribution of densities related to the molecule) only those images where the molecules are in the same orientation need to be averaged.

After scanning several negatives, several thousand images of single particles will be obtained, which is too many and will be too complex to be inspected and analysed visually. Multivariate statistical classification (MSA) can be used to analyse the huge number of images and group them into 'characteristic views' (Borland \& van Heel, 1990; Frank et al., 1981; van Heel, 1984; van Heel \& Stöffler-Meilicke, 1985). A combination of reference-free alignment and multivariate statistical analysis followed by 'multi-reference alignment' (MRA) procedures (Dube et al., 1993) allows optimization of the global centring and angular alignment of the data set. MSA data compression and automatic classification procedures (Borland \& van Heel, 1990; Frank et al., 1981; van Heel, 1989) are then used to sort images of molecules that have similar angular orientations into individual groups. These averages (classes) present molecular 'characteristic views'.

Our experience has shown that an adequate enhancement of the SNR to allow for reliable definition of the orientation of the characteristic view (see below) can be achieved if the average contains at least 15-20 images. Therefore, we can make a simple estimation of the number of images needed to obtain a 3D reconstruction at certain resolution (for example $\sim 5 \AA$ ) according to (Crowther et al., 1970)

$$
\operatorname{Res}=\pi D / K,
$$

where Res is the desired resolution, $D$ is the object size and $K$ is the number of different object views. For ribosomes, $D$ is $\sim 240 \AA$ and for $5 \AA$ resolution $K$ will be $\sim 150$. Therefore, to provide $\sim 20$ images per view the minimum number of raw images required is expected to be around 3000 . However, this is a very low estimate, since it assumes an equal probability of having projections in all directions. In reality, the distribution of projections around 
the Euler sphere can be rather heterogeneous. Although ribosomes are randomly distributed within the vitreous ice, some hydrophobic characteristics and surface charges of the molecule lead to preferred orientations in ice. Thus, some regions in the Euler sphere will be less populated. To compensate for the deficiency of projections in those directions, the number of projections has to be increased four to five times $(\sim 15000)$. This very simple assessment is consistent with other calculations (Henderson, 1995). Some groups are using much larger numbers, which may be a result of the different procedure of angle orientation determination.

\subsection{Orientation determination}

The previous step of image analysis has provided us with a set of different images, all having reliable 2D information (projections of an unknown 3D structure). At the beginning of the last century, a theorem stating that a distribution of densities from an object can be restored using $2 \mathrm{D}$ projections of the object was proved analytically by Radon (1917). However, this theorem assumes that angles between projections are known. Therefore, it is necessary to find the relative spatial orientation of the characteristic views.

There are several techniques at hand to solve this problem. One of them is a comparison of images with projections of a model in a range of directions. Assignment of the projection direction for each image is then made by identifying a particular model projection giving rise to the best match (Penczek et al., 1994; Baker \& Cheng, 1996). However, this approach requires an initial model. We use the 'angular reconstitution technique' (van Heel, 1987) to determine angular orientations of the characteristic views. The angular reconstitution method is based on the 'common-line projection' theorem stating that any two $2 \mathrm{D}$ projections of a $3 \mathrm{D}$ object have at least one common one-dimensional (1D) line projection. The same idea can be rephrased for Fourier space: the Fourier transforms of 2D projections intersect each other at the line passing through the origin of Fourier space. This line will be a common line for both Fourier transforms (Crowther et al., 1970). Its Fourier transform corresponds to a common $1 \mathrm{D}$ projection. The search for common lines in Fourier space was developed for icosahedral viruses, which have the highest symmetry of single particles (Crowther, 1971; Crowther et al., 1970).

The general idea of angular reconstitution is to compare sets of $1 \mathrm{D}$ projections from $2 \mathrm{D}$ projections and to find the common lines between pairs of projections. The angular distance between common lines of two projections with respect to a third will give the angle between those two projections. Analysis of the cross correlation of 1D projections of several views helps to refine their angular orientation. The number of common lines depends on the symmetry of the object. For an asymmetrical object such as the ribosome, any two projections will have only one common line. Symmetrical objects have several common lines (Orlova \& Heel, 1994). For example, keyhole limpet haemocyanin type 1 has $D_{5}$ symmetry and reveals ten common lines (for details, see Orlova et al., 1997).

\subsection{D reconstruction}

Having assigned angles to a set of projections, the 3D density map can be calculated. The major techniques for determination include direct Fourier transform methods, convolution algorithms and algebraic formulations. These have been described in several books (Frank, 1996; Herman, 1980). The Fourier-Bessel inversion method, introduced into EM by Crowther (Crowther, 1971; Crowther et al., 1970) and further developed by other groups (Baker \& Cheng, 1996; Baker et al., 1988; Fuller, 1987; Fuller et al., 1996), is an established technique used in the structural analysis of viruses. The exact-filter back-projection algorithm (Harauz \& van Heel, 1986; Radermacher, 1988) performs calculation of 3D maps in real space. This algorithm automatically downweights over-represented orientations (projections) in the data set.

\subsection{Iterative refinement}

As soon as the first reconstruction is obtained, a refinement can be performed as an iterative procedure. Projections of the first reconstruction are well centred and hence can be used as new references in the procedure of raw data alignment. More accurate alignment improves classification of images. Consequently, the orientations of the 'better classes' can be determined more accurately, resulting in an improved 3D structure. Reprojections from the improved 3D map can then be used as an anchor to refine the search for common lines (Serysheva et al., 1995). A complete cycle of image processing of single particles is presented in Fig. 1.

\subsection{Quality criteria}

How can one estimate the reliability of the obtained reconstruction? The quality of the $3 \mathrm{D}$ reconstruction depends on the variance of the images forming a class and the accuracy of the orientation determination. The results of the quality assessment of classification and the angle search lead to a decision on how to proceed. The input data set can be realigned to refine parameters such as the coordinates of image centres or the orientation angles found for characteristic views. The number of images or characteristic views used in the $3 \mathrm{D}$ reconstruction can also be changed, another cycle of iteration can be made or the procedure can be stopped and the result interpreted. To make the decision, the raw data used in processing can be compared with simulated data derived from the resulting model.

The final assessment of the 3D reconstruction quality reveals the size of the smallest reliable details (resolution) usually used for interpretation of the obtained 3D map. The assessment can be made by comparison of two 3D maps obtained from two subsets of the data by calculating the differential phase residual or the cross-correlation coefficients between the two corresponding shells in Fourier space (Frank et al., 1981; Saxton \& Baumeister, 1982; van Heel \& Harauz, 1986). These are more common approaches to estimate the resolution of a structure in EM analysis. Recently, a new method was suggested which overcomes the requirement to divide the data set in two. The method is based on assessment 
of spectral-signal-to-noise ratio in the obtained 3D reconstruction (Unser et al., 1996). A more interesting approach would be a comparative analysis of the results obtained from different techniques such as reconstructions using the projection matching and the angular reconstitution or the $\mathrm{X}$-ray structure and EM structure.

\section{Interpretation analysis of the structural features: structural analysis of the ribosome}

We applied the scheme of processing described above to the analysis of cryo-EM images of 50S ribosomal subunits obtained in our Philips CM200 FEG microscope at liquidnitrogen temperature. The images were taken at $200 \mathrm{kV}$. The excellent stability of the microscope and high coherence of the illuminating beam allowed us to obtain high-quality images even at the relatively high defocus used $(1.0-2.0 \mu \mathrm{m})$.

The E. coli $50 \mathrm{~S}$ ribosomal subunit structure at $7.5 \AA$ resolution (Matadeen et al., 1999) was obtained using 16000 particles from seven micrographs. These micrographs were digitized using the image Science patchwork densitometer and a final sampling frequency of $1.76 \AA \mathrm{pixel}^{-1}$ was used for the analysis. Multi-reference alignment procedures together with MSA, angular reconstruction and the exact-filter backprojection algorithm were used to obtain a 3D reconstruction ( $\sim 1100$ classes). The model was iteratively refined yielding the $7.5 \AA$ structure (Fig. 1). Resolution assessment was made using the Fourier shell correlation criterion and $3 \sigma$ threshold (three standard deviations of the random noise; van Heel \& Harauz, 1986).

The $7.5 \AA$ resolution structure shows the classical features of the 50S subunit, including the central protuberance (CP), the A-site finger (ASF), the L7/L12 stalk and the L1 protuberance (Fig. 2). This higher resolution has enabled us to
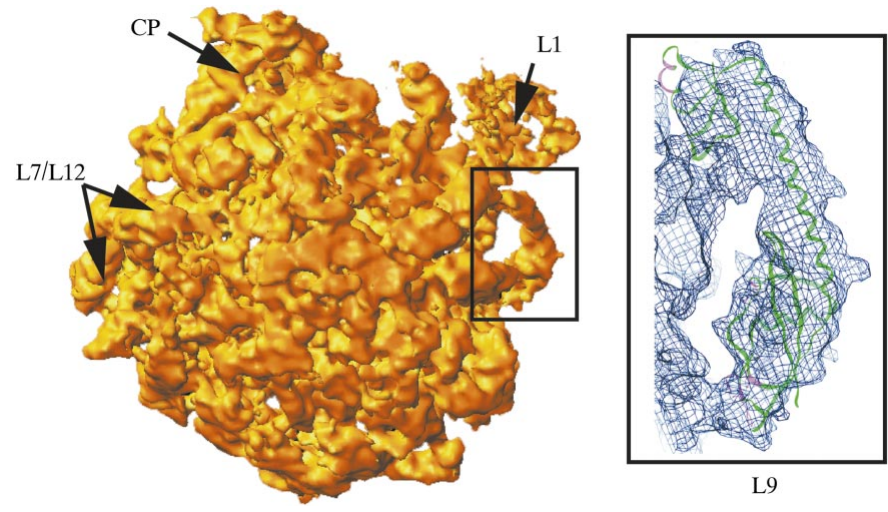

Figure 2

A view from the L1 side of the $50 \mathrm{~S}$ subunit. The L1 stalk is wrapped by the L9 protein, the N-terminal domain of which docks onto the L1 stalk directly. The long hydrophilic $\alpha$-helix connecting the $\mathrm{N}$ - and the C-terminal domains of L9 is seen here to be fully solvent exposed. On the right-hand side, fitting of the Bacillus stearothermophilus L9 protein (PDB code 1div) into the 3D density of the collar structure around the L1 stalk of the $7.5 \AA$ E. coli $50 \mathrm{~S}$ large ribosomal subunit cryo-EM reconstruction. The coordinates were fitted interactively into the $\mathrm{C}$ terminal domain (lower domain) in the cryo-EM density using the program $O$ (Jones et al., 1991).

observe novel features of the ribosome. A prominent new detail in the isolated 50S subunit is the collar-like structure around the L1 stalk (Fig. 2a). The X-ray/NMR structure of the L9 ribosomal protein (Hoffman et al., 1996) fits into this collar structure (Fig. 2). Protein L9 had previously been localized under the L1 protuberance by immuno-electron microscopy (Stöffler-Meilicke et al., 1983).

During the initiation phase of protein synthesis, the $50 \mathrm{~S}$ ribosomal subunit associates with a $30 \mathrm{~S}$ subunit complexed to fMet-tRNA $^{\mathrm{fMet}}$ (Stark et al., 2000). Consequently, the large subunit undergoes a conformational change upon interaction with the small subunit. These changes are the most apparent on the side of the isolated 50S subunit that faces the $30 \mathrm{~S}$ subunit within the $70 \mathrm{~S}$ complex (subunit interface side). In contrast, the whole solvent side of the 50S subunit map shows no major differences with respect to the bound 50S particle (Matadeen et al., 1999). The subunit interface side of the large subunit is relatively flat. However, on this surface there is a protrusion called the 'primary interface protrusion' (PIP; Fig. 3). This rRNA hairpin loop, which is involved in subunit association (Merryman et al., 1999), penetrates deep into the $30 \mathrm{~S}$ subunit within the $70 \mathrm{~S}$ complex.

\section{Conclusions}

Cryo-electron microscopy is becoming a standard tool for elucidating the structure of biological macromolecules. The technique affords the advantage of allowing the visualization of biologically different functional states. The current limitations include the resolutions achievable and the smallest size of particle analysable. It is here where both X-ray analysis and NMR can complement electron microscopy by providing highly resolved structural components of macromolecules or their assemblies, allowing the interpretation of functional movements of the components with greater precision. On the other hand, EM can assist X-ray analysis by providing initial phase information required for more intriguing biological

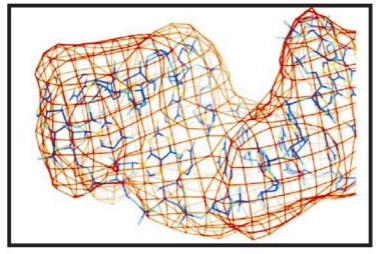

PIP

Figure 3

The current $7.5 \AA$ map has revealed the characteristic shape of an rRNA hairpin loop. Thus, we now believe the PIP probably represents the h34 helix, which was placed somewhat further back in our 23S rRNA model. On the left side, the small box locates a position of the PIP on the surface of the $50 \mathrm{~S}$ subunit. 
complexes. It therefore seems that the synergistic use of all these techniques will eventually lead to the elucidation of many more intriguing biological molecules.

The author would like to thank Mr R. Matadeen, Mr B. Gowen and Drs M. Cuff, T. Pape and M. van Heel for stimulating discussions. Ms M. Morris is acknowledged for editorial assistance. The work was in part financed by European Commission grants BIO4-CT97-2188 and BI04CT96-0592. The farm of Compaq EV6 computers used for alignment refinements was financed by the BBSRC Centre of Structural Biology grant 28/SB 11262. The CM-200/FEG microscope was funded by BBSRC/HEFCE Joint Research Equipment Initiative grant (JREI-97-8010).

\section{References}

Akiba, T., Toyoshima, C., Matsunaga, T., Kawamoto, M., Kubota, T., Fukuyama, K., Namba, K. \& Matsubara, H. (1996). Nature Struct. Biol. 3, 553-561.

Baker, T. S. \& Cheng, H. (1996). J. Struct. Biol. 116, 120-130.

Baker, T. S., Drak, J. \& Bina, M. (1988). Proc. Natl Acad. Sci. USA, 85, 422-426.

Borland, L. \& van Heel, M. (1990). J. Opt. Soc. Am. A, 7, 601-610.

Böttcher, B., Wynne, S. A. \& Crowther, R. A. (1997). Nature (London), 386, 88-91.

Boublik, M., Hellmann, W. \& Kleinschmidt, A. K. (1977). Cytobiology, 14, 293-300.

Chiu, W. (1993). Annu. Rev. Biophys. Biomol. Struct. 22, 233-255.

Conway, J. F. \& Steven, A. C. (1999). J. Struct. Biol. 128, 106-118.

Cramer, H. (1946). Mathematical Methods of Statistics. Princeton University Press.

Crowther, R. A. (1971). Philos. Trans. R. Soc. London Ser. B, 261, 221-230.

Crowther, R. A., DeRosier, D. J. \& Klug, A. (1970). Proc. R. Soc. London, 317, 319-340.

Dube, P., Tavares, P., Lurz, R. \& van Heel, M. (1993). EMBO J. 12, 1303-1309.

Dubochet, J., Adrian, M., Chang, J. J., Homo, J. C., Lepault, J., McDowall, A. W. \& Schultz, P. (1988). Q. Rev. Biophys. 21, 129228.

Dubochet, J., Lepault, J., Freeman, R., Berriman, J. A. \& Homo, J. C. (1982). J. Microsc. 128, 219-237.

Erickson, H. P. \& Klug, A. (1970). Philos. Trans. R. Soc. London Ser. $B, \mathbf{2 6 1}, 105-118$.

Frank, J. (1973). Optik, 38, 519-536.

Frank, J. (1996). Three-Dimensional Electron Microscopy of Macromolecular Assemblies. San Diego, London: Academic Press.

Frank, J., Radermacher, M., Wagenknecht, T. \& Verschoor, A. (1988). Methods Enzymol. 164, 3-35.

Frank, J., Verschoor, A. \& Boublik, M. (1981). Science, 214, 13531355.

Frank, J., Verschoor, A., Boublik, M. \& van Heel, M. (1982). J. Mol. Biol. 161, 107-137.

Fujiyoshi, Y. (1999). FASEB J. 13 Suppl. 2, 191-194.

Fuller, S. D. (1987). Cell, 48, 923-934.

Fuller, S. D., Butcher, S. J., Cheng, R. H. \& Baker, T. S. (1996). J. Struct. Biol. 116, 48-55.

Glaeser, R. M. (1985). Annu. Rev. Phys. Chem. 36, 243-275.

Harauz, G. \& van Heel, M. (1986). Optik, 73, 146-156.

Harris, J. R. \& Horne, R. W. (1991). Electron Microscopy in Biology, edited by J. R. Harris, pp. 203-228. Oxford: IRL Press.
Hawkes, P. W. T. (1992). In Electron Tomography: Three-Dimensional Imaging with the Transmission Electron Microscope, edited by J. Frank. New York, London: Plenum Press.

Heel, M. van (1984). Ultramicroscopy, 13, 165-184.

Heel, M. van (1987). Ultramicroscopy, 21, 111-124.

Heel, M. van (1989). Optik, 82, 114-126.

Heel, M. van \& Frank, J. (1981). Ultramicroscopy, 6, 187-194.

Heel, M. van \& Harauz, G. (1986). Optik, 73, 119-122.

Heel, M. van \& Stöffler-Meilicke, M. (1985). EMBO J. 4, 2389-2395.

Henderson, R. (1995). Quart. Rev. Biophys. 28, 171-193.

Henderson, R., Baldwin, J. M., Downing, K. H., Lepault, J. \& Zemlin, F. (1986). Ultramicroscopy, 19, 147-178.

Herman, G. T. (1980). Image Reconstruction from Projections: the Fundamentals of Computerized Tomography. New York: Academic Press.

Hoffman, D. W., Cameron, C. S., Davies, C., White, S. \& Ramakrishnan, V. (1996). J. Mol. Biol. 264, 1058-1071.

Hoppe, W. \& Hegerl, R. (1980). Computer Proccesing of Electron Microscope Images, edited by P. W. Hawkes, pp. 127-185. Berlin, New York: Springer-Verlag.

Jones, T. A., Zou, J.-Y., Cowan, S. W. \& Kjeldgaard, M. (1991). Acta Cryst. A47, 110-119.

Lake, J. A. (1982). J. Mol. Biol. 161, 89-106.

Lamvik, M. K. (1991). J. Microsc. 161, 171-181.

Ludtke, S. J., Baldwin, P. R. \& Chiu, W. (1999). J. Struct. Biol. 128, 8297.

Luther, P. K., Lawrence, M. C. \& Crowther, R. A. (1988). Ultramicroscopy, 24, 7-18.

Matadeen, R., Patwardhan, A., Gowen, B., Orlova, E., Pape, T., Cuff, M., Mueller, F., Brimacombe, R. \& van Heel, M. (1999). Structure, 7, 1575-1583.

Merryman, C., Moazed, D., Daubresse, G. \& Noller, H. F. (1999). J. Mol. Biol. 285, 107-113.

Mitsuoka, K., Murata, K., Walz, T., Hirai, T., Agre, P., Heymann, J. B., Engel, A. \& Fujiyoshi, Y. (1999). J. Struct. Biol. 128, 34-43.

Miyazawa, A., Fujiyoshi, Y., Stowell, M. \& Unwin, N. (1999). J. Mol. Biol. 288, 765-786.

Nogales, E., Wolf, S. G. \& Downing, K. H. (1997). J. Struct. Biol. 118, 119-127.

Orlova, E. V., Dube, P., Harris, J. R., Beckman, E., Zemlin, F., Markl, J. \& van Heel, M. (1997). J. Mol. Biol. 271, 417-437.

Orlova, E. V. \& van Heel, M. (1994). 13th International Congress of Electron Microscopy, Vol. 1, edited by B. Jouffrey \& C. Collix, pp. 507-508. Les Ulis, Paris: Les Editions de Physique.

Penczek, P. A., Grassucci, R. A. \& Frank, J. (1994). Ultramicroscopy, 53, 251-270.

Radermacher, M. (1988). J. Electr. Microsc. Tech. 9, 359-394.

Radermacher, M., Wagenknecht, T., Verschoor, A. \& Frank, J. (1987). EMBO J. 6, 1107-1114.

Radon, J. (1917). Math. Phys. Klasse, 69, 262-277.

Saxton, W. O. (1978). Computer Techniques for Image Processing in Electron Microscopy, p. 289. New York, San Francisco, London: Academic Press.

Saxton, W. O. \& Baumeister, W. (1982). J. Microsc. 127, 127-138.

Serysheva, I. I., Orlova, E. V., Chiu, W., Sherman, M. B., Hamilton, S. L. \& van Heel, M. (1995). Nature Struct. Biol. 2, 18-24.

Stark, H., Rodnina., M. V., Wieden, H.-J., van Heel, M. \& Wintermeyer, W. (2000). Cell, 100, 301-309.

Stark, H., Zemlin, F. \& Boettcher, C. (1996). Ultramicroscopy, 63, 7679.

Stöffler-Meilicke, M., Noah, M. \& Stöffler, G. (1983). Proc. Natl Acad. Sci. USA, 80, 6780-6784.

Unser, M., Trus, R. L., Vrehl, M. J., Conway, J. F., Gross, M., Thevenaz, R. \& Steven, A. C. (1996). Resolution Assessment of Three-Dimensional Reconstructions by Spectral Signal-to-Noise Ratio. In Abstracts of the 11th European Congress on Electron Microscopy. Dublin: U. C. D. Belfield. 